\title{
Effect of dietary modifications on the cholesterol level and selected indicators of oxidative processes in rats with mammary cancer
}

\author{
Tomasz Lepionka ${ }^{1,2}$, Agnieszka Bialek ${ }^{2}$, Malgorzata Bialek ${ }^{3}$, Marian Czauderna ${ }^{3}$, \\ Jerzy Bertrandt ${ }^{1}$, Anna Anyzewska ${ }^{1}$, Roman Lakomy ${ }^{1}$ and Barbara Bobrowska-Korczak ${ }^{2}$ \\ ${ }^{1}$ Laboratory of Food and Nutrition Hygiene, Military Institute of Hygiene and Epidemiology, Warsaw, Poland, \\ ${ }^{2}$ Department of Bromatology, Medical University of Warsaw, Warsaw, Poland and \\ ${ }^{3}$ The Kielanowski Institute of Animal Physiology and Nutrition, Polish Academy of Sciences, Warsaw, Poland
}

\begin{abstract}
Introduction: Pomegranate (Punica granatum, Punicaceae) seed oil (PSO) and bitter melon (Momordica charantia, Cucurbitaceae) water extract (BME), used for centuries in folk medicine, are now available as popular dietary supplements, used i.e. in prevention of diet-related metabolic diseases and cancer. It is suggested that their mechanism of action may involve modifications of oxidative processes. However, information regarding these effects is inadequate.
\end{abstract}

Aim: This study aimed to evaluate the influence of supplementation of rats' diet with PSO and BME, administered separately or jointly, on cholesterol and its oxidative derivatives, malonyldialdehyde (MDA) and tocopherols content in livers, in terms of cancerous process. Sprague-Dawley female rats $(\mathrm{n}=96)$ were fed control diet $(\mathrm{CON}$ and CONplus) or experimental diets supplemented with $0.15 \mathrm{ml}$ PSO/day (G and Gplus), 1\% BME ad libitum (M and Mplus) and PSO and BME jointly (GM and GMplus). Breast cancer was induced by administration of 7,12-dimethylbenz[a]anthracene (DMBA) in the amount of $80 \mathrm{mg} / \mathrm{kg}$ body weight in the $50^{\text {th }}$ day of life. After 21 weeks of supplementation livers, as main organs responsible for lipids metabolism, were subjected to either GC-TOFMS (cholesterol and oxysterols) or UPLC-PDA (tocopherols and MDA) chromatographic analyzes.

Results and discussion: Both dietary supplements and DMBA administration resulted in significant increase of total cholesterol content. In contrast, levels of two of four determined oxysterols: $7 \alpha$ - hydroxycholesterol and cholesterol $\beta$-epoxide were the lowest in groups supplemented with PSO and exposed to DMBA. Significant differences in tocopherol content were demonstrated for two of four detected compounds: g-tocopherol and $\alpha$-tocopheryl acetate. The level of g-tocopherol in $\mathrm{G}$ group was significantly higher in relation to Mplus and Gplus groups. In case of $\alpha$-tocopheryl acetate, differences were observed between two control groups (CON and CONplus), but its content in CONplus group was significantly higher. In physiological state applied supplements reduced MDA levels in livers, whereas in pathological conditions PSO significantly increased MDA content in hepatic tissue of Gplus and GMplus groups.

Conclusions: The obtained results indicate that applied dietary supplementation, as well as cancerous process, can modify the levels of cholesterol and intensity of oxidative processes. It seems, that oxidative stress, associated with cancer development, modifies the intensity of oxidative processes in livers to a greater extent than examined dietary supplements.

* This work was partially supported by National Science Centre, Poland (grant number 2018/02/X/NZ9/01770)

\section{Conflict of Interest}

There is no conflict of interest 